\title{
Focal Values of Plane Cubic Centers
}

\author{
Hans-Christian Graf v. Bothmer • Jakob Kröker
}

Received: 21 February 2009 / Accepted: 9 July 2010 / Published online: 14 October 2010

(C) The Author(s) 2010. This article is published with open access at Springerlink.com

\begin{abstract}
We prove that the vanishing of 11 focal values is not sufficient to ensure that a complex plane cubic system has a complex center. This is done by finding a complex cubic system with a high order weak focus using an extensive computer search.
\end{abstract}

\section{Introduction}

In 1885 Poincaré asked when the differential equation

$$
y^{\prime}=-\frac{x+p(x, y)}{y+q(x, y)}=:-\frac{P(x, y)}{Q(x, y)}
$$

with convergent power series $p(x, y)$ and $q(x, y)$ starting with quadratic terms, has stable solutions in the neighborhood of the equilibrium solution $(x, y)=(0,0)$. This means that in such a neighborhood the solutions of the equivalent plane autonomous system

Supported by the German Research Foundation [Deutsche Forschungsgemeinschaft (DFG)] through the Institutional Strategy of the University of Göttingen.

H.-C. Graf v. Bothmer $(\varangle)$ J J. Kröker

Courant Research Centre "Higher Order Structures", Mathematisches Institiut, Georg-August-Universität Göttingen, Bunsenstrasse 3-5, 37073 Göttingen, Germany e-mail: bothmer@uni-math.gwdg.de

URL: http://www.crcg.de/wiki/Bothmer

\section{J. Kröker}

e-mail: kroeker@uni-math.gwdg.de

URL: http://www.crcg.de/wiki/User:Kroeker 


$$
\begin{aligned}
& \dot{x}=y+q(x, y)=Q(x, y) \\
& \dot{y}=-x-p(x, y)=-P(x, y)
\end{aligned}
$$

are closed curves around $(0,0)$.

Poincaré showed that one can iteratively find a formal power series

$$
F=x^{2}+y^{2}+f_{3}(x, y)+f_{4}(x, y)+\cdots
$$

such that

$$
\operatorname{det}\left(\begin{array}{cc}
F_{x} & F_{y} \\
P & Q
\end{array}\right)=\sum_{j=1}^{\infty} s_{j}\left(x^{2 j+2}+y^{2 j+2}\right)
$$

with $s_{j}$ rational polynomials in the coefficients of $P$ and $Q$. If all $s_{j}$ vanish and $F$ is convergent, then $F$ is a constant of motion, i.e. its gradient field satisfies $P d x+Q d y=0$. Since $F$ starts with $x^{2}+y^{2}$ this shows that close to the origin all integral curves are closed and the system is stable. Therefore the $s_{j}$ 's are called the focal values of $P d x+Q d y$. Often also the notation $\eta_{2 j}:=s_{j}$ is used, and the $\eta_{i}$ are called Lyapunov quantities.

Poincaré also showed, that if an analytic constant of motion exists, the focal values must vanish. Later Frommer [2] proved that the systems above are stable if and only if all focal values vanish even without the assumption of convergence of $F$. (Frommer's proof contains a gap which can be closed [10]).

Unfortunately it is in general impossible to check this condition for a given differential equation because there are infinitely many focal values. In the case where $P$ and $Q$ are polynomials of degree at most $d$, the $s_{j}$ are rational polynomials in finitely many unknowns. Hilbert's Basis Theorem then implies that the ideal $I_{\infty}=\left(s_{1}, s_{2}, \ldots\right)$ is finitely generated, i.e there exists an integer $m:=m(d)$ such that

$$
s_{1}=s_{2}=\cdots=s_{m(d)}=0 \Longrightarrow s_{j}=0 \quad \forall j .
$$

This shows that a finite criterion for stability exists, but due to the indirect proof of Hilbert's Basis Theorem no value for $m(d)$ is obtained. In fact even today only $m(2)=$ 3 is known. Żołądek [11] and Christopher [1] showed that $m(3) \geq 11$. Since the number of variables for $d=2$ is six and $m(2)=6-3$ it has been conjectured that for $d=3$ with 14 variables one has $m(3)=14-3=11$. Notice that the minimal $m(d)$ can depend on whether complex or real coefficients are considered. It is the purpose of this note to prove $m(3) \geq 12$ for complex plane systems.

The most naive approach to this problem is to calculate a Gröbner Basis of $I_{11}=$ $\left(s_{1}, \ldots, s_{11}\right)$ and prove that $s_{12} \notin I_{11}$ by the usual ideal membership test. Unfortunately this is not feasible, since the $s_{j}$ have very many terms and huge coefficients. In the case of cubic $P$ and $Q$ they involve 14 variables and are of weighted degree $2 j$. For example the $s_{6}$ calculated with our version of Frommer's Algorithm [7] has already 95,760 terms. 
Żołądek and Christopher therefore deduce their result geometrically. They exhibit a component $Y_{11} \subset X_{\infty}=V\left(I_{\infty}\right)$ that has codimension 11 in the space of all possible $(P, Q)$ of degree at most three. Finding a component of codimension 12 is not an easy task, and indeed we choose a different approach. We prove that there exist a codimension 11 family complex plane autonomous system of degree 3 which have a non integrable saddle and for which nevertheless the first 11 focal values vanish, but 12 th one doesn't. For this we look at the system

$$
\begin{aligned}
& \dot{x}=y+3 x^{2}+8 x y+5 y^{2}+3 x^{3}+25 x^{2} y+20 x y^{2}+18 y^{3} \\
& \dot{y}=-\left(x+27 x^{2}+9 x y+22 y^{2}+11 x^{3}+20 x^{2} y+4 x y^{2}+3 y^{3}\right)
\end{aligned}
$$

and prove that for this system $s_{j}=0 \bmod 29$ for $j \leq 11$ while $s_{12} \neq 0 \bmod 29$. Checking that furthermore the Jacobian matrix of $s_{1}, \ldots, s_{11}$ has full rank modulo 29 for this system, we can apply a theorem of Schreyer [5] to show the existence of the desired family of foci over $\mathbb{C}$. From this we deduce that $s_{12} \notin I_{11}=\left(s_{1}, \ldots, s_{11}\right)$. In fact we even prove the stronger result $s_{12} \notin \operatorname{rad} I_{11}$.

Since for a given system one can evaluate the $s_{j}$ using Frommers algorithm [7] without knowing the complete polynomials, this approach is feasible.

We found the above system by performing a random search. Heuristically each $s_{i}$ vanishes mod 29 for about one of every 29 differential equations [9]. So we expect to find an example as above after checking $29^{11} \approx 10^{16}$ random examples. By parameterizing $s_{1}$ and $s_{2}$ we can improve this to $29^{9} \approx 10^{13}$ random examples. Indeed we found the example after about $8 \times 10^{12}$ trials. Using an improved version [8] of the program [6] this took 1,246 CPU-days. Since this search is easily parallelizable we could do this calculation in about one month by distributing the work to 12 AMD Opteron Dual Core computers with $2.2 \mathrm{GHz}, 128 \mathrm{~K}$ L1-cache and 1024K L2-cache and 30 Intel Pentium 4 computers with $3 \mathrm{GHz}, 32 \mathrm{~K} \mathrm{L1}$-cache and 2048K L2-cache. The program uses only $5 \mathrm{MB}$ of RAM.

We would like to thank the Regionales Rechenzentrum für Niedersachsen (RRZN) and the Institut für Systems Engineering, Fachgebiet Simulation for providing the necessary CPU time. Also we are grateful to Colin Christopher who checked our example using REDUCE [4].

\section{The Proof}

Notation 2.1 If $I \subset \mathbb{Z}\left[x_{1}, \ldots, x_{n}\right]$ is an ideal and $X_{\mathbb{Z}}=V(I) \subset \mathbb{A}_{\mathbb{Z}}^{n}$ is the variety over spec $\mathbb{Z}$ defined by $I$, then we denote by $X_{\mathbb{F}_{p}}$ the fiber of $X_{\mathbb{Z}}$ over $\mathbb{F}_{p}$ for any prime $p$. Furthermore we denote by $X_{\mathbb{C}}$ the variety defined by $I$ over $\mathbb{C}$.

Theorem 2.2 (Schreyer) Let $I=\left(f_{1}, \ldots, f_{k}\right) \subset \mathbb{Z}\left[x_{1}, \ldots, x_{n}\right]$ be an ideal and $X_{\mathbb{Z}}=V(I)$. If $x \in X_{\mathbb{F}_{p}}$ is a point with codim $T_{X_{\mathbb{F}}, x}=k$ then there exists an irreducible component $Y_{\mathbb{Z}} \subset X_{\mathbb{Z}}$ with $x \in Y_{\mathbb{Z}}$ and $Y_{\mathbb{Z}} \not \subset X_{\mathbb{F}_{p}}$. In particular $Y_{\mathbb{C}} \neq \emptyset$.

Proof This is a special case of a theorem of Schreyer [5]. See also [3] for a proof.

Example 2.3 Consider $X_{\mathbb{Z}}=V(3 x) \subset \mathbb{A}_{\mathbb{Z}}^{1}$. This variety has two components over $\mathbb{Z}$ namely $Y_{\mathbb{Z}}=V(x)$ and $Z_{\mathbb{Z}}=V(3)$. Since $3=0$ is true only in $\mathbb{F}_{3}$ we have 


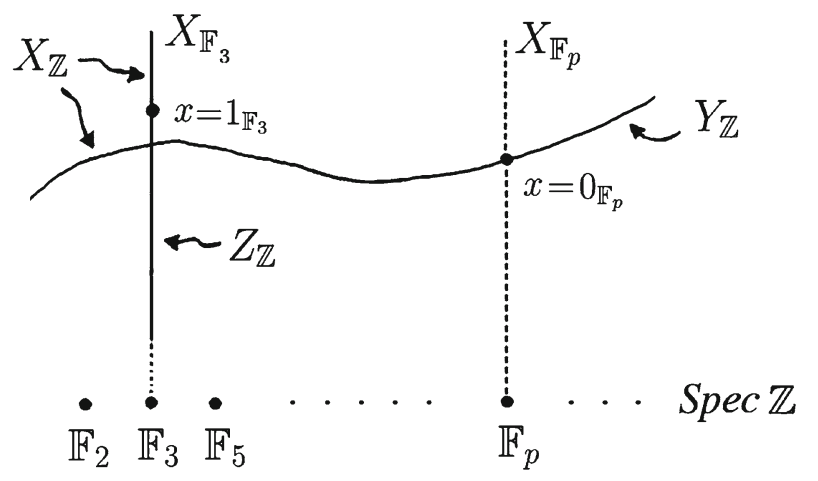

Fig. 1 A variety over $\operatorname{spec} \mathbb{Z}$

$Z_{\mathbb{Z}}=Z_{\mathbb{F}_{3}}$. Furthermore $Z_{\mathbb{C}}=\emptyset$. On the other hand $x=0$ is possible over all $\mathbb{F}_{p}$ and $Y_{\mathbb{C}} \neq \emptyset$. See Fig. 1 .

Indeed, if we consider the point $x=0 \in X_{\mathbb{F}_{p}}, p \neq 3$ then we have that the derivative $(3 x)^{\prime}=3 \neq 0$ and the tangent space $T_{0, X_{\mathbb{F}}}$ has codimension 1 . Therefore the theorem applies and the component $Y_{\mathbb{Z}}$ containing $x=0_{\mathbb{F}_{p}}$ is not contained in $X_{\mathbb{F}_{p}}$.

Since $3 \cdot 1=0 \in \mathbb{F}_{3}$ we can also consider the point $x=1 \in X_{\mathbb{F}_{3}}$. Here we have $(3 x)^{\prime}=3=0$ and the tangent space $T_{1, X_{\mathbb{F}_{3}}}$ has codimension 0 . Hence the theorem does not apply, and indeed the component $Z_{\mathbb{Z}}=Z_{\mathbb{F}_{3}}$ containing $x=1_{\mathbb{F}_{3}}$ is completely contained in $X_{\mathbb{F}_{3}}$.

Corollary 2.4 If in the situation of Theorem 2.2 we have a further polynomial $g \in$ $\mathbb{Z}\left[x_{1}, \ldots, x_{n}\right]$ satisfying $g(x) \neq 0 \in \mathbb{F}_{p}$ then $g$ does not vanish on $X_{\mathbb{C}}$.

Proof Assume to the contrary that $g$ vanishes on $X_{\mathbb{C}}$. By Theorem 2.2 we have a component $Y_{\mathbb{Z}} \subset X_{\mathbb{Z}}$ with $x \in Y_{\mathbb{Z}}$ and $Y_{\mathbb{C}} \neq \emptyset$. Since $g$ vanishes on $X_{\mathbb{C}}$ and $Y_{\mathbb{C}} \neq \emptyset$ it also vanishes on $Y_{\mathbb{C}}$ and therefore on $Y_{\mathbb{Z}}$ and $Y_{\mathbb{F}_{p}}$. But this contradicts our assumption $g(x) \neq 0$.

Example 2.5 Notice that conditions of Theorem 2.2 and Corollary 2.4 do not imply the existence of real points $x \in X_{\mathbb{C}}$ with $g(x)=0$. Consider for example the family of plane systems

$$
\begin{aligned}
& \dot{x}=-y+a x^{2}+a x y \\
& \dot{y}=x-b x y+b y^{2} .
\end{aligned}
$$

In this case

$$
\begin{aligned}
& s_{1}=\frac{1}{8}\left(a^{2}+b^{2}\right) \\
& s_{2}=\frac{1}{6} a^{3}(3 a-4 b) .
\end{aligned}
$$

For $a=2$ and $b=1$ we have $s_{1} \equiv 0(5)$ and $s_{2} \not \equiv 0(5)$. Since the Jacobian matrix in this case has the correct rank, Corollary 2.4 implies that for certain $a$ and $b$ over the 
complex numbers we have a complex focus of order 2. Over the real numbers however this family of systems never has a focus of order 2 , since $s_{1}=0$ implies $a=b=0$ and therefore also $s_{2}=0$. This example was kindly provided by the referee.

Theorem $2.6 m(3) \geq 12$ for complex plane systems.

Proof Using our implementation of Frommers algorithm [7,6,8] we obtain

$$
\begin{aligned}
& s_{1}=145=5 \cdot 29 \\
& s_{2}=\frac{-2810738}{5}=\frac{(-1) \cdot 2 \cdot 7^{2} \cdot 23 \cdot 29 \cdot 43}{5} \\
& s_{3}=\frac{112175678099}{60}=\frac{29 \cdot 6163 \cdot 627637}{2^{2} \cdot 3 \cdot 5} \\
& s_{4}=\frac{-4609839516974492}{567}=\frac{(-1) \cdot 2^{2} \cdot 29 \cdot 4914541 \cdot 8086207}{3^{4} \cdot 7} \\
& s_{5}=\frac{15087652045867048479893}{311850}=\frac{29 \cdot 10181 \cdot 51101450117924357}{2 \cdot 3^{4} \cdot 5^{2} \cdot 7 \cdot 11} \\
& s_{6}=\frac{-47800111276327585549868098577}{125096400} \\
& =\frac{(-1) \cdot 7 \cdot 29 \cdot 43 \cdot 61 \cdot 89770693272899615845933}{2^{4} \cdot 3^{7} \cdot 5^{2} \cdot 11 \cdot 13} \\
& s_{7}=\frac{122592296723640794761191530195327149667}{31786995240000} \\
& =\frac{29 \cdot 761 \cdot 9261610935427 \cdot 599782781246472744509}{2^{6} \cdot 3^{8} \cdot 5^{4} \cdot 7 \cdot 11^{3} \cdot 13} \\
& s_{8}=\frac{-998199449131240887417361925153989295656095071273}{20766229924745930625} \\
& =\frac{(-1) \cdot 23 \cdot 29 \cdot 8111 \cdot 649787 \cdot 83993087 \cdot 110599614673 \cdot 30566725375870417}{3^{11} \cdot 5^{4} \cdot 7^{3} \cdot 11^{4} \cdot 13^{3} \cdot 17} \\
& s_{9}=\frac{145010763456597069697037235091337410694847561167439689383417}{202215898592636341533120000} \\
& =\frac{29 \cdot 241 \cdot 8233 \cdot 64381 \cdot 4901740681 \cdot 1228805547453217}{2^{9} \cdot 3^{11} \cdot 5^{4} \cdot 7^{4} \cdot 11^{5} \cdot 13^{4} \cdot 17 \cdot 19} . \\
& .6498840374505787565393 \\
& s_{10}=\frac{(-1) \cdot 29 \cdot 291640957}{2^{8} \cdot 3^{14} \cdot 5^{5} \cdot 7^{6} \cdot 11^{5} \cdot 13^{5} \cdot 17^{3} \cdot 19} . \\
& \text {.3688454531370071629111510617021416082954922488168734079515731 } \\
& s_{11}=\frac{29 \cdot 47 \cdot 321467 \cdot 804541 \cdot 121836860182878614056682214997}{2^{11} \cdot 3^{16} \cdot 5^{5} \cdot 7^{6} \cdot 11^{5} \cdot 13^{6} \cdot 17^{4} \cdot 19^{3} \cdot 23} . \\
& \cdot 1858776194792400195182106117486816782933 \\
& s_{12}=\frac{(-1) \cdot 2099 \cdot 409261 \cdot 74003353 \cdot 2724272714124740629841}{2^{6} \cdot 3^{17} \cdot 5^{7} \cdot 7^{7} \cdot 11^{6} \cdot 13^{6} \cdot 17^{5} \cdot 19^{4} \cdot 23} . \\
& .550524948891154683105490668924575639203339371258291071
\end{aligned}
$$

This shows that the first 11 focal values are divisible by 29 while the 12 th is not. Similarily one can check that the codimension of the tangent space to the variety 
cut out by the first 11 focal polynomials in this point over $\mathbb{F}_{29}$ is 11 . Therefore the conditions of Theorem 2.2 and Corollary 2.4 are satisfied.

Note added in proof After submitting this article we have found the following example

$$
\begin{aligned}
& \dot{x}=y+8 x^{2}+27 x y+12 y^{2}+27 x^{3}+14 x^{2} y+9 x y^{2}+13 y^{3} \\
& \dot{y}=-\left(x+27 x^{2}+24 x y+18 y^{2}+20 x^{3}+28 x^{2} y+20 x y^{2}+25 y^{3}\right)
\end{aligned}
$$

by extending our computer search. For this example the first 12 focal values vanish modulo 31, but the 13th one does not. Also the rank of the Jacobi-matrix of $s_{1}, \ldots, s_{12}$ has full rank modulo 31 . From Theorem 2.2 and Corollary 2.4 it then follows that even $m(3) \geq 13$ for complex systems.

Open Access This article is distributed under the terms of the Creative Commons Attribution Noncommercial License which permits any noncommercial use, distribution, and reproduction in any medium, provided the original author(s) and source are credited.

\section{References}

1. Christopher, C.: Estimating limit cycle bifurcations from centers. In Differential Equations With Symbolic Computation, Trends Math., pp. 23-35. Birkhäuser, Basel (2005)

2. Frommer, M.: Über das Auftreten von Wirbeln und Strudeln (geschlossener und spiraliger Integralkurven) in der Umgebung rationaler Unbestimmtheitsstellen. Math. Ann. 109, 395-424 (1934)

3. Graf v. Bothmer, H.-C.: Cord Erdenberger, and Katharina Ludwig. A new family of rational surfaces in $\mathbb{P}^{4}$. J. Symbolic Comput. 39(1), 51-60 (2005)

4. Hearn, A.: REDUCE. Available at http://www.reduce-algebra.com (2004)

5. Schreyer, F.-O.: Small fields in constructive algebraic geometry. In Moduli of vector bundles (Sanda, 1994; Kyoto, 1994). Lecture Notes in Pure and Appl. Math., vol. 179, pp. 221-228. Dekker, New York (1996)

6. Graf v. Bothmer, H.-C., Martin, C.: A C++ program for calculating focal values in characteristic $p$. Available at http://www-ifm.math.uni-hannover.de/ bothmer/strudel (2005)

7. Graf v. Bothmer, H.-C., Martin, C.: Frommers algorithm. NoDEA 14(5-6), 694-698 (2007)

8. Graf v. Bothmer, H.-C., Kröker, J.: A improved C++ program for calculating focal values in characteristic $p$. Available at http://www.stud.uni-hannover.de/ kroeker/centerfocus/index.html or at http:// sourceforge.net/projects/centerfocus/ (2009)

9. Graf v. Bothmer, H.-C., Schreyer, F.-O.: A quick and dirty irreducibility test for multivariate polynomials over $\mathbb{F}_{q}$. Experiment. Math. 14(4), 415-422 (2005)

10. Wolf v. Wahl.: Personal communication (2005)

11. Żołądek, H.: Eleven small limit cycles in a cubic vector field. Nonlinearity 8(5), 843-860 (1995) 